\title{
Immune reconstitution inflammatory syndrome associated with pulmonary sarcoidosis in an HIV-infected patient: an immunohistochemical study
}

\begin{abstract}
Sarcoidosis has been rarely described in literature as a cause of interstitial pulmonary disease associated with AIDS. This study reports a case of immune reconstitution inflammatory syndrome associated with pulmonary sarcoidosis in a patient with a history of previous pulmonary tuberculosis concomitant with HIV infection. Results of the immunohistochemical study of samples from the resected right lower lobe are described. Pathological findings suggest a role of Th1, Th2 and Th17 response in IRIS associated sarcoidosis.
\end{abstract}

Keywords: acquired immunodeficiency syndrome; sarcoidosis, pulmonary; immune reconstitution inflammatory syndrome; immunohistochemistry.

\section{INTRODUCTION}

Sarcoidosis has been rarely described in literature as a cause of interstitial pulmonary disease associated with AIDS. The majority of cases have been related to immune restoration secondary to highly active antiretroviral therapy (HAART) in human immunodeficiency virus (HIV) infected patients. ${ }^{1}$ Association between CD4+ T lymphocyte count higher than 200 cells $/ \mathrm{mm}^{3}$ and worsening of sarcoidosis course, as well as association between CD4+ less than 200 cells $/ \mathrm{mm}^{3}$ and improvement of symptoms have been demonstrated in the literature. ${ }^{2}$

The clinical and pathological features of sarcoidosis in HIV-infected patients are similar to HIV-uninfected patients and have been reported after use of IL-2 or IFN-alpha therapy in both HIVinfected patients and HIV-uninfected persons. ${ }^{3}$ Probably, immune reconstitution is associated with an immune dysregulation that induces the Th1 immune responses against unknown antigens related to sarcoidosis. ${ }^{3}$

This is a diagnosis of exclusion, since several interstitial diseases, including granulomatous inflammation, occur in HIV-infected patients, such as pulmonary disease due to Mycobacterium spp., Pneumocystis jiroveci, Cryptococcus spp., Toxoplasma gondii, cytomegalovirus, Kaposi's sarcoma, pulmonary nonspecific interstitial disease and lymphoid interstitial pneumonitis. ${ }^{1,3}$

Immune reconstitution inflammatory syndrome (IRIS) may be defined as a paradoxical exacerbation of symptoms of opportunistic infections, inflammatory diseases or tumours, in patients responding to antiretroviral therapy (ART), with decrease in plasma HIV RNA level by $1 \log _{10}$ copies/mL. ${ }^{4}$ Minor criteria include increased blood CD4+ T cell after ART and improved specific immune response directed to a relevant pathogen or epitope.

IRIS can induce sarcoid-like lesions in tissues within the first few weeks of initiating therapy. The pathogenesis of these granulomasiscurrently unknown because no pathogen has been identified to date in the lesions using morphological and/or microbiological approaches. ${ }^{5}$ However, the role of certain microbes, such as Mycobacterium tuberculosis, is still controversial in literature. ${ }^{5}$

This study reports a case of pulmonary sarcoidosis related to IRIS in a patient with a previous history of pulmonary tuberculosis and concomitant HIV infection and describes the results of the immunohistochemical evaluation performed in the resected lower lobe of the right lung of this patient. Informed consent was obtained from patient after explaning the study.
Authors

Érique José Peixoto de Miranda $^{1}$

Olavo Henrique Munhoz Leite $^{2}$

Maria Irma Seixas

Duarte $^{3}$

${ }^{1} \mathrm{MD}$, Resident Physician, Department of Infectious Diseases, Medical School, Universidade de São Paulo (USP), SP, Brazil ${ }^{2}$ Assistant Physician, Department of Infectious Diseases, Medical School, USP, Brazil

${ }^{3}$ Professor, Department of Pathology, Medical School, USP, Brazil

Submitted on: 01/25/2011 Approved on: 03/17/2011

Correspondence to:

Érique José Peixoto de Miranda

Enéas de Carvalho

Aguiar, 255

Cerqueira Cesar

05403-000

São Paulo, SP

Brazil

eriquemiranda@gmail.com

We declare no conflict of interest.

(C2011 Elsevier Editora Ltda. All rights reserved. 


\section{MATERIALS AND METHODS}

A search with the terms "sarcoidosis" and "HIV" and "AIDS" was performed in PubMed, from 1966 to 2010, in order to find reported cases of this association. The data obtained was summarized by statistical analysis using the software SPSS 10.0 for Windows (SPSS Statistics IBM, Chicago, IL, USA).

Fragments of lung from the patient described in this study were stained by hematoxylin-eosin, Ziehl-Neelsen, Grocott and immunohistochemistry for Bacillus Camette-Guérin (BCG) from formalin-fixed, paraffin-embedded specimens.

Immunohistochemistry was performed in order to characterize the composition of inflammatory cells in granulomas with an indirect streptavidin-biotin complex immunoperoxidase technique on a DAKO TechMate (DAKO, Glostrup, Denmark).

The following antibodies were employed: anti-CD20, CD14, CD4, CD8, CD14, CD57, CD68, toll like receptor 2 (TLR-2), TLR-4, TLR-9, C3, FoxP3, TCRab, TNF-a, IFN-g, IL-1b, IL-2R, IL-4, IL-5, IL-6, IL-8, IL-10, IL-18, TGFb, and S100 (Dako, Glostrup, Denmark). Evaluation was performed in 10 high power fields (i.e. field of vision at a magnification of 1:400). The color intensity was controlled with an optical microscope using positive controls that accompany the reaction. Omission of the primary antibodies served as negative control. Times with washing buffer and incubations were followed strictly according to the manufacturer's instructions.

\section{CASE REPORT}

A 29-year-old white male patient with a history of AIDS and pulmonary tuberculosis, diagnosed two years ago was admitted. The patient complained of daily evening fever ranging from $38^{\circ} \mathrm{C}$ to $38.5^{\circ} \mathrm{C}$, productive cough, weight loss of $10 \mathrm{~kg}$ (normal was $70 \mathrm{~kg}$ ) and progressive dispnea in the past six months. These clinical features began seven months after starting antiretroviral therapy with zidovudine, lamivudine and efavirenz, on a regular basis.

Previous diagnosis of tuberculosis was based only in a positive sample of sputum for alcohol acid-fast bacilli (Ziehl-Neelsen technique) showing 1 to 10 bacilli per field in 50 examinedfields. Hehad been treated with isonyazid $400 \mathrm{mg}$, rifampin $600 \mathrm{mg}$ and pyrazinamide 2,000 $\mathrm{mg}$ daily during two months followed by isonyazid $400 \mathrm{mg}$ plus rifampin $600 \mathrm{mg}$ daily during four months and was discharged after having a negative sputum for alcohol acid-fast bacilli and improvement of symptoms at the end of treatment.

CD4+ $\mathrm{T}$ cell count before starting antiretroviral therapy was 234 cells $/ \mathrm{mm}^{3}$ and plasma HIV-1 RNA was 34,564 copies/mL (branched-DNA technique), seven months before onset of symptoms. At admission, CD4+ count was 618 cells $/ \mathrm{mm}^{3}$ and plasma HIV-1 RNA was undetectable $(<50$ copies $/ \mathrm{mL})$. Despite the immune restoration, the patient presented three episodes of right lower lobe community acquired pneumonia, one of them complicated with empyema, within eight months before hospital admission.

$\mathrm{He}$ also reported previous heterosexual exposure risk and past consumption of $80 \mathrm{~g}$ of alcohol per day for 11 years, but stated to have stopped drinking four years before .

At admission, he had no changes on physical examination except for dyspnoea at rest, requiring nasal oxygen catheter at a flow rate of $2 \mathrm{~L} / \mathrm{min}$, digital clubbing and watch glass nails and auscultation with diminished breath sounds in the right pulmonary apex. There were no skin lesions. Arterial blood gas collected at room air revealed $\mathrm{pH} 7.26$, $\mathrm{pO}_{2}=50.8 \mathrm{mmHg}, \mathrm{pCO}_{2}=53.1 \mathrm{mmHg}, \mathrm{SO}_{2}: 79.3 \%, \mathrm{HCO}_{3}$ : $23.7 \mathrm{mmol} / \mathrm{L}$, base excess $(\mathrm{BE}):+3.9 \mathrm{mmol} / \mathrm{L}$. Lactate dehydrogenase was $398 \mathrm{IU} / \mathrm{L}$ (normal range $[\mathrm{NR}]<480 \mathrm{IU} / \mathrm{L}$ ) and C-reactive protein was $3.16 \mathrm{mg} / \mathrm{L}(\mathrm{NR}<5 \mathrm{mg} / \mathrm{L})$.

Assessment of delayed-type hypersensitivity response to tuberculin by a skin test turned out zero millimeter (response absent). Three samples of sputum and brochoalveolar lavage (BAL) were negative for mycobacteria and fungi in direct examination and culture.

Thoracic computed tomography $(\mathrm{CT})$ scan revealed bilateral pleural thickening, bilateral lung volume reduction in upper lobes, parenchymal distortion, bronchiectasis and thin-walled multiloculated cavities mainly in superior segment of the right lower lobe, diffuse areas of ground glass opacities in both lungs, as well as slight mediastinal paratracheal and subcarinal lymphadenopathy of about $1.3 \mathrm{~cm}$, and increased caliber of the pulmonary artery and its main branches, consistent with pulmonary hypertension (Figure 1). There was no signal of pulmonary fibrosis.

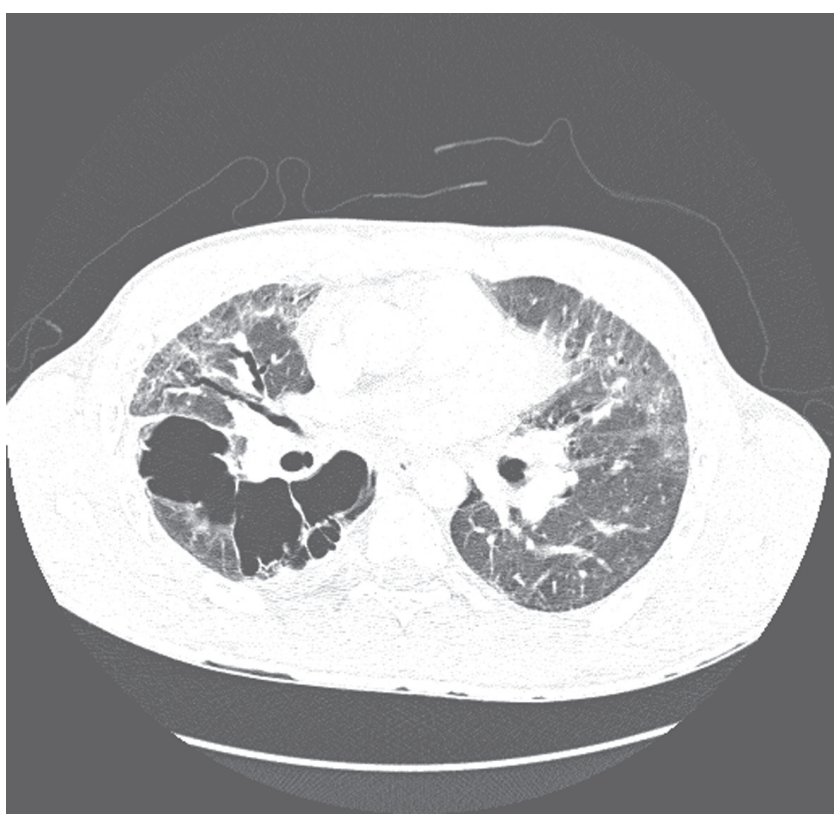

Figure 1: Chest CT revealed bilateral lung volume reduction due to upper lobes and parenchymal distortion, bronchiectasis and multiloculated cavities with thin walls over the upper lobes, and diffuse areas of ground glass opacities in both lungs. 
The electrocardiogram showed sinus rhythm, heart rate of $75 \mathrm{bpm}, \mathrm{P}$ and QRS waves with normal duration and voltages, normal QTc (400 ms) and signs of right ventricular overload probably due to arterial pulmonary hypertension: QRS axis of $+180^{\circ}$, S1Q3T3 pattern, $\mathrm{qR}$ in V1, increased S wave in V5-V6 and $\mathrm{rS}$ in V2-V6. The echocardiogram was unable to estimate the pulmonary artery pressure due to poor acoustic window.

The pulmonary function tests revealed severe restrictive defect: forced vital capacity (FVC) of $1.64 \mathrm{~L}$ (32\% predicted), forced expiratory volume in one second (FEV1): 1.37 L (33\%), FEV1/FVC: 84\%, and no improvement after bronchodilators.

The patient was submitted to a diagnostic and therapeutic thoracotomy and right lower lobe resection. Material obtained showed diffuse bronchiectasis. The pathology report showed scattered noncaseating granulomas forming clusters (sarcoid-like), with Langhans and foreign-body-type giant cells, around the bronchioles and vascular bundles, as well as areas of alveolitis, without evidence of Schaumann and asteroid bodies (Figure 2). Acid-fast stain and cultures for Mycobacterium spp. and fungi turned out negative.

Abdominal CT scan and central nervous system (CNS) magnetic resonance imaging revealed no evidence of sarcoidosis in the liver, spleen and CNS. Cerebrospinal fluid (CSF) analysis was also normal: one white blood cell per $\mathrm{mm}^{3}$ (55\% lymphocytes and $10 \%$ monocytes), protein: $39 \mathrm{mg} / \mathrm{dL}(\mathrm{NR}<45 \mathrm{mg} / \mathrm{dL})$ and glucose $80 \mathrm{mg} / \mathrm{dL}$ (NR: $50-90 \mathrm{mg} / \mathrm{dL}$ ). Gram and acidfast stain as well as bacterial and fungal culture were all negative. Urinary calcium was of $435.2 \mathrm{mg}$ /volume in

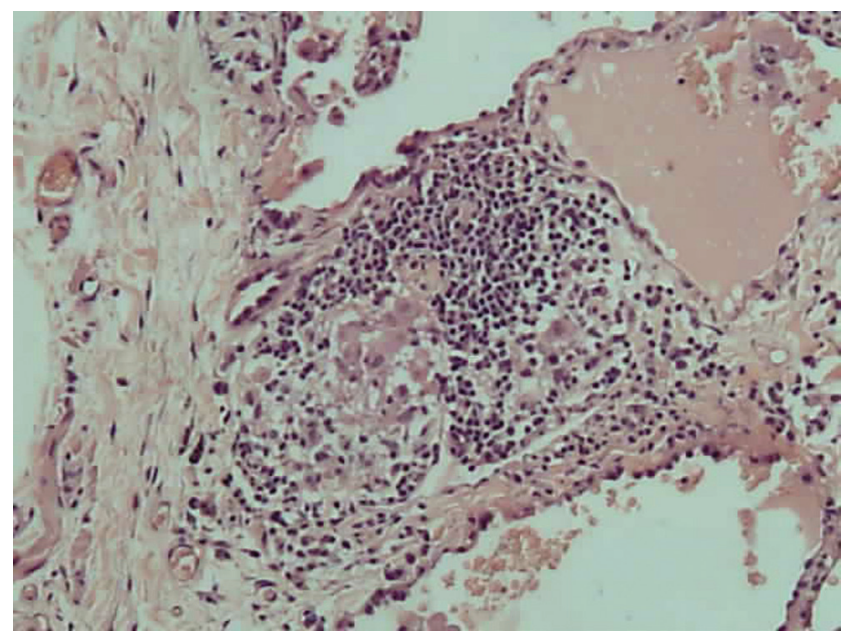

Figure 2: Hematoxylin-eosin staining of resected lung segment from the patient, 100x magnification: clustered noncaseating granuloma around the terminal airways and areas of alveolitis. Search for infectious agents resulted negative.
24 hours (NR: 100-320 mg). Serum ionized calcium was normal: $4.7 \mathrm{mg} / \mathrm{dL}$ (NR: 4.6 to $5.3 \mathrm{mg} / \mathrm{dL}$ ).

The patient was discharged with prednisone $30 \mathrm{mg}$ daily $(0.5 \mathrm{mg} / \mathrm{kg})$, home oxygen therapy and hydroxychloroquine $400 \mathrm{mg}$ daily due to hypercalciuria. Six months thereafter the patient improved his dyspnea and pulmonary function: a 30\% increase in FEV1 and FVC. A new thoracic CT three months after starting corticotherapy revealed reduction of thin-walled multiloculated cavities and diminished caliber of bronchiectasis in all lobes of both lungs, mainly in the left upper lobe. The response to corticosteroids and hydroxycloroquine was considered satisfactory. The patient continues to use the medication in outpatient setting and discontinued home oxygen therapy six months after.

\section{RESULTS}

Immunohistochemistry evaluation of the granulomas showed strong intensity of staining for IL-17 (Th17 response) (Figure 3), dendritic cells (S100), macrophages (CD68), CD4+ T cell, CD8+ T cell, B cell (CD20), TCR-alpha/beta, IL-2R, IL-4 (Figure 4), TGF-beta, IL-10 and IL-12. Immunohistochemistry for IL-1 (Figure 5), IL-6, IL-8 and TNF-alpha showed strong staining both in granulomas and in alveolitis. There was a predominance of CD4+ T cell in alveolitis. Toll-like receptor-2 (TLR-2), TLR-4 and C3 showed a strong staining, but TLR-9 showed weak staining, as well as CD57 (NK cells), IFN-gamma and forkhead-box transcription factor (FoxP3) both in granulomas and alveolitis (Figure 6). Grocott staining and immunohistochemistry for BCG were negative (Figures 7 and 8).

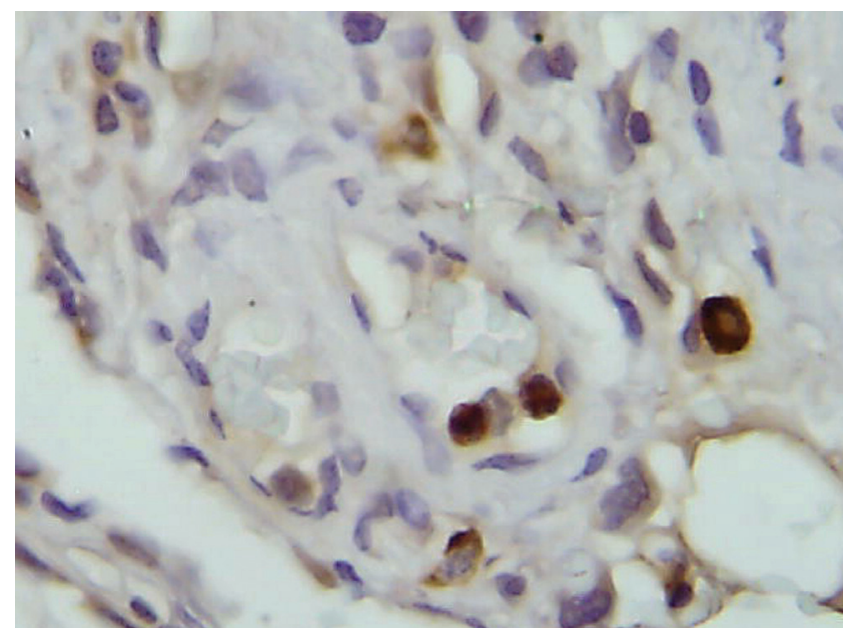

Figure 3: Immunohistochemistry for IL-17, marker of Th17 response, showed strong staining in granulomas and alveoli (400x magnification). 


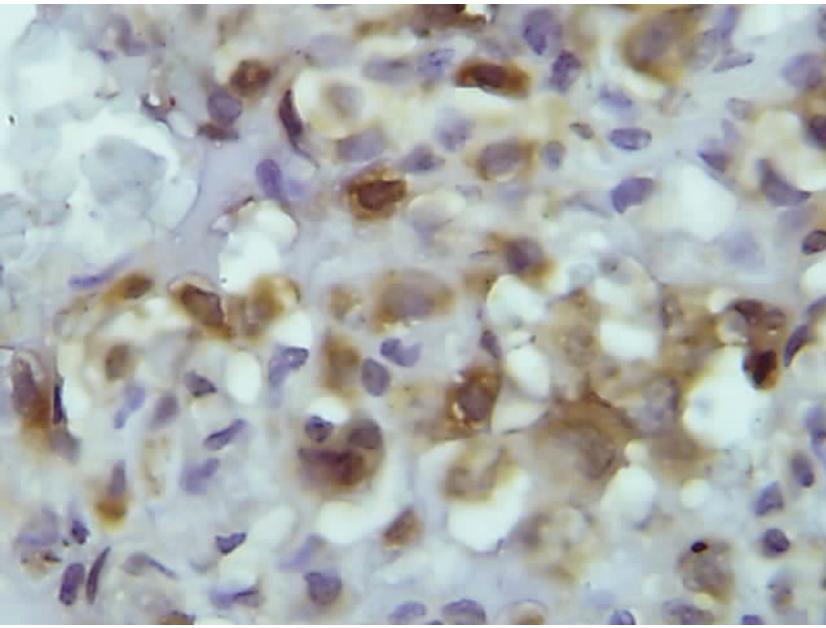

Figure 4: Immunohistochemistry for $\mathrm{IL}-4$, marker of Th2 response, showed strong staining in granulomas and alveoli (400x magnification).

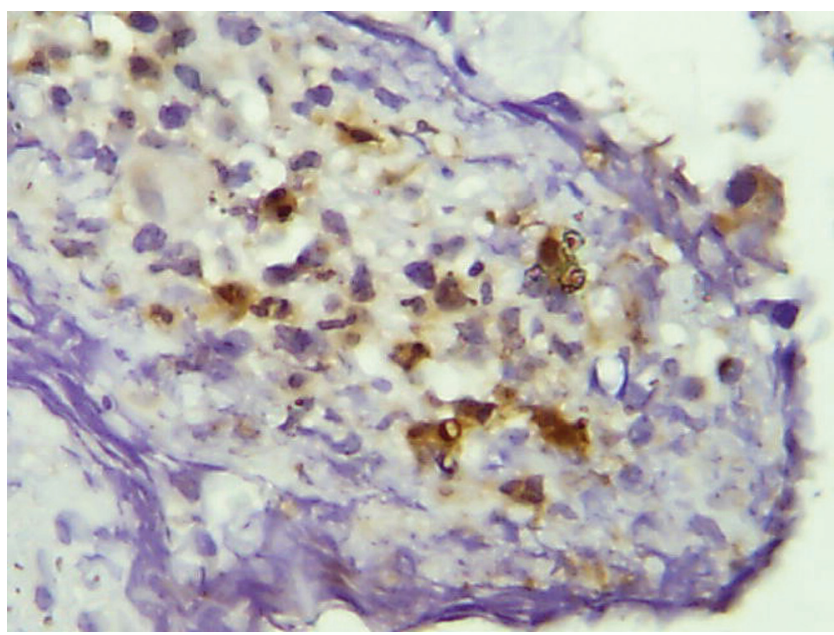

Figure 5: Immunohistochemistry for IL-1-beta, marker of Th1 response, revealed strong staining in the core of granulomas (400x magnification). This cytokine was also detected in alveolitis.

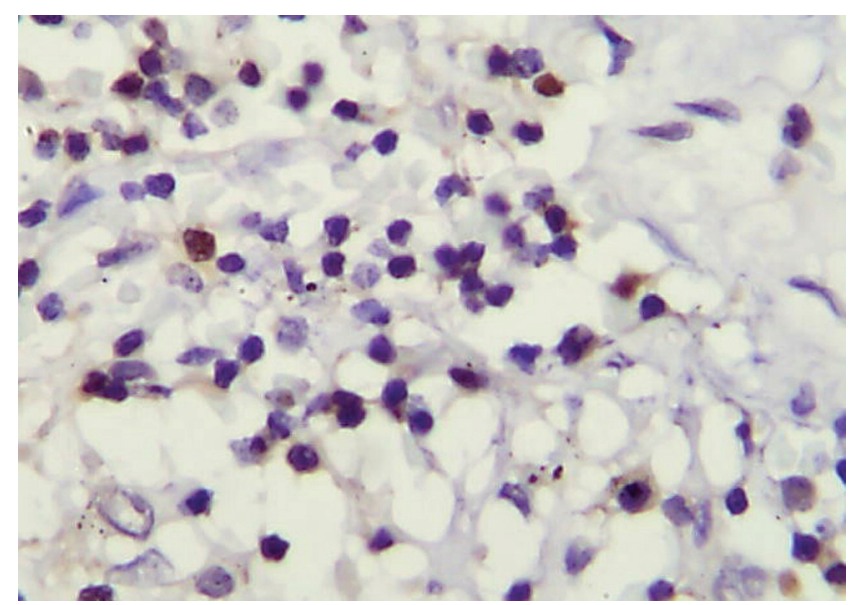

Figure 6: Immunohistochemistry for FoxP3, marker of Treg response, revealed weak staining in the core of granulomas (400x magnification).

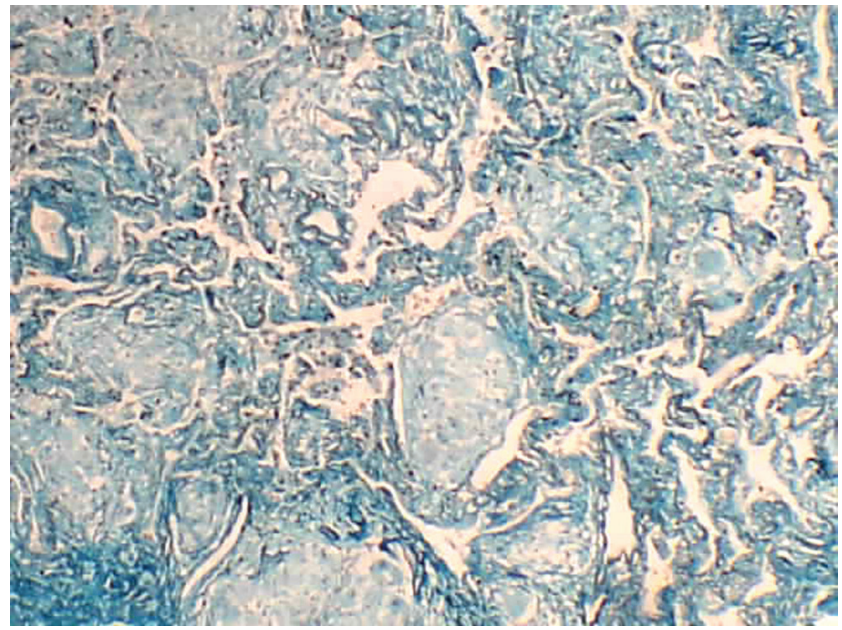

Figure 7: Grocott staining (400x magnification): search for fungi was negative.

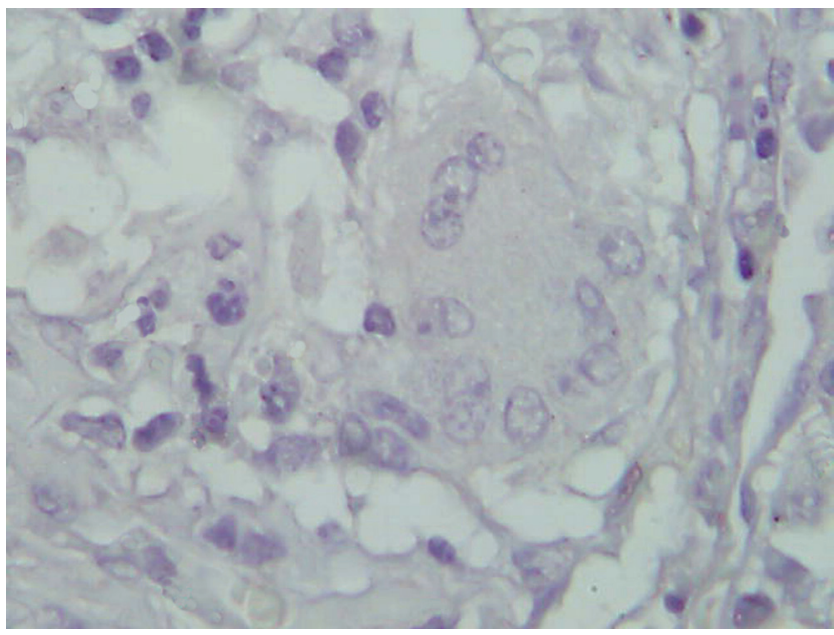

Figure 8: Immunohistochemistry for bacillus Calmette-Guerin (BCG) was negative (400x magnification).

\section{DISCUSSION}

This case presents a stage III pulmonary sarcoidosis in a white man, characterized by pulmonary hypertension and obstructive and restrictive ventilatory impairment, beginning seven months after the introduction of antiretroviral therapy. Thoracic CT scan showed an atypical pattern suggestive of alveolar filling, probably due to the IRIS. Clinical evolution characterized by good response to corticosteroids and repeated negative search for infectious agents reinforced this hypothesis.

There were 55 cases of AIDS and sarcoidosis association in international literature, 14 of those before the HAART era, based on a positive Kveim-Stilzbach test in AIDS patients. ${ }^{1,6-19}$ All cases ruled out infectious agents and demonstrated sarcoidosis by anatomopathological findings. 
Two of these patients presented the symptoms after therapy with IL-2. The median of time between antiretroviral therapy and onset of symptoms of sarcoidosis was 9 months (95\% CI: 5.63-13.19 months), ranging from 0 to 48 months, longer than IRIS associated with granulomatous infectious diseases (few weeks). The median age was 37 years (interquartile range [IR]: 12.5 years) and 59.1\% were male. The median CD4+ nadir before symptoms was 11 cells $/ \mathrm{mm}^{3}$ (IR: $197 / \mathrm{mm}^{3}$ ) and at onset of symptoms, CD4 count was 341 cells $/ \mathrm{mm}^{3}$ (IR: 272 cells $/ \mathrm{mm}^{3}$ ) ( $\mathrm{p}<0.05$, Wilcoxon test). Median highest plasma viral load before symptoms was 9,400 copies/mL (IR: 36,123 copies $/ \mathrm{mL}$ ) and during the event was $<50$ copies $/ \mathrm{mL}$ ( $\mathrm{p}<0.05$, Wilcoxon test). The CD4:CD8 ratio on broncoalveolar lavage was $3.37( \pm 3.01)$. Dispnea was the most frequent symptom $(32.7 \%)$, followed by constitutional symptoms (23.5\%), skin disease (13.7\%) and lymphadenopathy $(12 \%)$. Three patients were asymptomatic. Foulon et al., ${ }^{7}$ found abnormal pulmonary function test in four of 11 patients, with a restrictive pattern in three and an obstructive pattern in one. Clinicopathological features were similar between HIV-uninfected and HIV-infected patients. ${ }^{1,6-19}$

The radiological stage II of pulmonary sarcoidosis was the most frequent (33.3\%), followed by stage I (25.5\%) and stage III (15.7\%). The evolution was unfavorable in $7.8 \%$ of these patients, characterized by poor response to treatment. No death due to IRIS associated with sarcoidosis was described in the literature.

Haramati et al. ${ }^{16}$ described thoracic CT findings in eight patients with IRIS associated with sarcoidosis in HIV patients: peribronchovascular and subpleural nodules and mediastinal lymphadenopathy were the most frequent finding. Ground glass opacities were observed in four of these patients. No association between the radiographic features of sarcoidosis and the CD4+ cell count was found.

Data from the literature are similar to findings of this case. The disease presented a worsening of symptoms associated with restoration of immune response following antiretroviral therapy. The magnitude of immune restoration may be demonstrated by the difference between CD4+ counts both in the case described here and in 55 cases from the literature. Infectious agents and other causes of interstitial pulmonary disease were always ruled out.

This is the first study in literature of immunohistochemistry in an HIV patient with IRIS-associated sarcoidosis. Pathological findings suggest a role of Th1 and Th2 response and imbalance of Treg/Th17, as well as role of complement, TLR-2 and 4 (innate immunity), in the pathogenesis of IRIS associated with sarcoidosis in this patient.

IRIS is characterized by replenishment of immune cells depleted by HIV infection, circulating of immune cells situated in compartments of the immune system, regeneration of lymphoid organs and recovery of pathogen or antigenspecific T, B, and NK response, diversity of response and regulation of the reconstituted immune system. ${ }^{3}$ The antigen-specific Th1 (granulomatous inflammation) and Th17 (suppurative inflammation) response, augmented against mycobacteria, fungi and protozoans in the context of IRIS, ${ }^{3}$ is also associated to sarcoidosis in this case report. Described in literature as a response that causes genesis and perpetuation of the inflammatory response, ${ }^{20} \mathrm{Th} 17$ cytokines are depleted in progressive lentiviral disease, characterized by loss of Th17/Treg balance. ${ }^{21}$

However, the patient has reduced expression of FoxP3 in granulomas, probably a marker of a diminished role of Treg response in the tissue affected by IRIS associated with sarcoidosis. This response has been implicated as a cause of peripheral anergy, but in pulmonary granulomas it has been associated to remission of fibrosis. ${ }^{22,23}$ Shift Th1 to Th2 response in sarcoidosis is associated to progressive fibrosis, ${ }^{22,23}$ characteristic not found in this patient. It was demonstrated in literature this shift in the progression of HIV infection. ${ }^{24}$

Conflicting findings were the weak expression of IFNgamma in this immunohistochemical study and normal serum level of C-reactive protein, which is described in the literature as a marker and a predictor of IRIS. ${ }^{25}$ Further studies are needed to explain these findings.

\section{CONCLUSION}

Considering all the clinic, radiology, laboratory and pathology data, this case presents a well described immune-reconstitution associated with sarcoidosis and suggests a possible role of Th1 and Th17 response in this syndrome. Further studies with larger samples are needed to confirm and explain the pathophysiology of these findings.

\section{REFERENCES}

1. Almeida Jr FA, Sager JS, Eiger G. Coexistent sarcoidosis and HIV infection: an immunological paradox? J Infect. 2006; 52(3):195-201.

2. Morris DG, Jasmer RM, Huang L, et al. Sarcoidosis following HIV infection: evidence for CD4+ lymphocyte dependence. Chest. 2003;124(3):929-35.

3. French MA. Immune Reconstitution Inflammatory Syndrome: A Reappraisal. Clin Infect Dis. 2009; 48:101-7.

4. Lassalle S, Selva E, Hofman V, et al. Sarcoid-like lesions associated with the immune restoration inflammatory syndrome in AIDS: absence of polymerase chain reaction detection of Mycobacterium tuberculosis in granulomas isolated by laser capture microdissection. Virchows Arch. 2006;449(6):689-96.

5. French MA, Price P, Stone SF. Immune restoration disease after antiretroviral therapy. AIDS. 2004; 18(12):1615-1627.

6. Amin DN, Sperber K, Brown LK. Positive Kveim test in patients with coexistingsarcoidosis and human immunodeficiency virus infection. Chest. 1992;101;1454-1456. 
7. Foulon G, Wislez M, Naccache JM, et al. Sarcoidosis in HIVinfected patients in the era of highly active antiretroviral therapy. Clin Infect Dis. 2004: 38 (3): 418-425.

8. Viani RM. Sarcoidosis and interstitial nephritis in a child with acquired immunodeficiency syndrome: implications of immune reconstitution syndrome with an indinavir-based regimen. Pediatr Infect Dis J. 2002;21:435-8.

9. Naccache JM, Antoine M, Wislez M, et al. Sarcoid-like pulmonary disorder in human immunodeficiency virus-infected patients receiving antiretroviral therapy. Am J Respir Crit Care Med. 1999;159:2009-13.

10. Blanche P, Gombert B, Rollot F, Salmon D, Sicard D. Sarcoidosis in a patient with acquired immunodeficiency syndrome treated with interleukin-2. Clin Infect Dis. 2000;31:1493-4.

11. Morris DG, Jasmer RM, Huang L, Gotway MB, Nishimura S, King TE Jr. Sarcoidosis following HIV infection: evidence for CD4+ lymphocyte dependence. Chest. 2003;124:929-35.

12. Trevenzoli M, Cattelan AM, Marino F, Marchioro U, Cadrobbi P. Sarcoidosis and HIV infection: a case report and a review of the literature. Post Med J. 2003;79(935):535-8.

13. Ferrand RA, Cartledge JD, Connolly J, Standish RA, Miller RF. Immune reconstitution sarcoidosis presenting with hypercalcaemia and renal failure in HIV infection. Int J STD \& AIDS. 2007; 18: 138-139.

14. Shelburne SA $3^{\text {rd }}$, Hamill RJ, Rodriguez-Barradas MC, et al. Immune reconstitution inflammatory syndrome emergence of a unique syndrome during highly active antiretroviral therapy. Medicine. 2002; 81(3): 213-27.

15. Lenner R, Bregman Z, Teirstein AS, DePalo L. Recurrent pulmonary sarcoidosis in HIV-infected patients receiving highly active antiretroviral therapy. Chest. 2001; 119: 978-81.
16. Haramati LB, Lee G, Singh A, Molina PL, White CS. Newly diagnosed pulmonary sarcoidosis in HIV-infected patients. Radiology. 2001; 218: 242-6.

17. Gomez V, Smith PR, Burack J, Daley R, Rosa U. Sarcoidosis after antiretroviral therapy in a patient with acquired immunodeficiency syndrome. Clin Infect Dis. 2000; 31: 1278-80.

18. Mirmirani P, Maurer TA, Herndier B, McGrath M, Weinstein MD, Berger TG. Sarcoidosis in a patient with AIDS: A manifestation of immune restoration syndrome. J Am Acad Dermatol. 1999; 41: 285-6.

19. Blanche P, Passeron A, Gombert B, et al. Sarcoidosis and HIV infection: influence of highly active antiretroviral therapy. $\mathrm{Br} J$ Dermatol. 1999; 140: 1185.

20. Korn T, Bettelli E, Oukka M, Kuchroo VK. IL-17 and Th17 Cells. Ann Rev Immunol. 2009; 27: 485-517.

21. Kanwar B, Favre D, McCune JM. Th17 and regulatory T cells: implications for AIDS pathogenesis. Curr Opin HIV AIDS. 2010; 5:151-157.

22. Iannuzzi MC, Rybicki BA, Teirstein AS. Sarcoidosis. N Engl J Med. 2007;357 (21):2153-65.

23. Ziegenhagen MW, Müller-Quernheim J. The cytokine network in sarcoidosis and its clinical relevance. J Intern Med. 2003, 253: 18-30.

24. Klein SA, Dobmeyer JM, Dobmeyer TS, et al. Demonstration of the Th1 to Th2 cytokine shift during the course of HIV-1 infection using cytoplasmic cytokine detection on single cell level by flow Cytometry. AIDS. 1997, 11:1111-1118.

25. Sereti I, Rodger AJ, French MA. Biomarkers in immune reconstitution inflammatory syndrome: signals from pathogenesis. Curr Opin HIV AIDS. 2010, 5:504-510. 